\title{
Reduction of radiation biases by incorporating the missing cloud variability by means of downscaling techniques: a study using the 3-D MoCaRT model
}

\author{
S. Gimeno García ${ }^{1,2}$, T. Trautmann ${ }^{1}$, and V. Venema ${ }^{3}$ \\ ${ }^{1}$ DLR - German Aerospace Center, Remote Sensing Technology Institute, Oberpfaffenhofen, 82234 Weßling, Germany \\ ${ }^{2}$ TUM - Technical University of Munich, 80333 München, Germany \\ ${ }^{3}$ University of Bonn, 53012 Bonn, Germany \\ Correspondence to: S. Gimeno García (sebastian.gimenogarcia@dlr.de)
}

Received: 2 January 2012 - Published in Atmos. Meas. Tech. Discuss.: 14 February 2012

Revised: 6 July 2012 - Accepted: 9 July 2012 - Published: 20 September 2012

\begin{abstract}
Handling complexity to the smallest detail in atmospheric radiative transfer models is unfeasible in practice. On the one hand, the properties of the interacting medium, i.e., the atmosphere and the surface, are only available at a limited spatial resolution. On the other hand, the computational cost of accurate radiation models accounting for three-dimensional heterogeneous media are prohibitive for some applications, especially for climate modelling and operational remote-sensing algorithms. Hence, it is still common practice to use simplified models for atmospheric radiation applications.

Three-dimensional radiation models can deal with complex scenarios providing an accurate solution to the radiative transfer. In contrast, one-dimensional models are computationally more efficient, but introduce biases to the radiation results.

With the help of stochastic models that consider the multifractal nature of clouds, it is possible to scale cloud properties given at a coarse spatial resolution down to a higher resolution. Performing the radiative transfer within the cloud fields at higher spatial resolution noticeably helps to improve the radiation results.

We present a new Monte Carlo model, MoCaRT, that computes the radiative transfer in three-dimensional inhomogeneous atmospheres. The MoCaRT model is validated by comparison with the consensus results of the Intercomparison of Three-Dimensional Radiation Codes (I3RC) project.

In the framework of this paper, we aim at characterising cloud heterogeneity effects on radiances and broadband
\end{abstract}

fluxes, namely: the errors due to unresolved variability (the so-called plane parallel homogeneous, PPH, bias) and the errors due to the neglect of transversal photon displacements (independent pixel approximation, IPA, bias). First, we study the effect of the missing cloud variability on reflectivities. We will show that the generation of subscale variability by means of stochastic methods greatly reduce or nearly eliminate the reflectivity biases. Secondly, three-dimensional broadband fluxes in the presence of realistic inhomogeneous cloud fields sampled at high spatial resolutions are calculated and compared to their one-dimensional counterparts at coarser resolutions. We found that one-dimensional calculations at coarsely resolved cloudy atmospheres systematically overestimate broadband reflected and absorbed fluxes and underestimate transmitted ones.

\section{Introduction}

Clouds are the most complex objects of the Earth's atmosphere. However, their shape, extension and degree of inhomogeneity greatly depend on the cloud type. For instance, strongly convective clouds (e.g., cumulonimbus) are highly inhomogeneous, whereas boundary layer clouds (e.g., marine stratocumulus) appear to be nearly homogeneous. In broken cloudy skies, the radiation intensity is decreased by cloud blocking, but also enhanced by reflection on cloud sides, which leads to alternating shaded and extra illuminated regions on the surface. 
Earth's average cloud fraction is about two thirds (Rossow and Zhang, 1995; Maddux et al., 2010), so a considerable part of the incoming and outgoing radiation is affected by clouds while traversing the atmosphere. This fact lends clouds a distinguished place in the Earth's radiation system: Clouds are recognised to be the main regulators of the radiation energy budget and, therefore, they are among the atmospheric constituents that affect climate and weather most. Moreover, clouds are a principal concern in remote-sensing applications. Since the interaction of clouds with radiation is complex, observations contaminated with clouds are usually avoided when retrieving atmospheric molecular concentrations and surface properties. Further, even when clouds are the main goal of the observations, one-dimensional (1D) approximate radiative transfer (RT) codes are used in the retrieval models.

Handling complexity to the smallest detail in RT models is in practice unfeasible. Two main reasons prevent this: the optical properties of the Earth's atmosphere and surface are not available at an arbitrarily high resolution and timeconsuming accurate models for solving the radiative transfer in three-dimensional resolved media are prohibitive for some applications, especially for climate modelling and operational remote-sensing algorithms. Additionally, in some cases, the use of simplified models is justified because they deliver an exact solution and this has advantages in the inversion theory.

Three-dimensional (3-D) radiation models can account for much more complexity than one-dimensional (1-D) ones providing a more accurate solution of the radiative transfer at the cost of forsaking the exact analytical solution and increasing considerably the calculation time.

Continuous technology progress has led to an increase of computing power, therefore, more sophisticated models can be used, e.g., for radiative transfer computations (e.g., Evans, 1998; Barker et al., 2003; Buras and Mayer, 2011). Accordingly, many three-dimensional models have been developed to study cloud variability and its multi-fractal structure (e.g., Venema et al., 2006; Watson et al., 2009; Lovejoy et al., 2009; Bar-Or et al., 2011). Furthermore, quantity and quality of input data will be significantly improved with the launch of the Sentinel satellites in the framework of the European Global Monitoring for Environment and Security (GMES) programme. So, the actual situation offers a perfect scenario to test the adequacy of the 1-D radiative transfer theory and opens the possibility to explore alternatives.

Stochastic models that combine cloud information at different spatial resolutions can be used to scaling the variability of cloud properties at a large range of scales. Hence, the synergistic use of the cloud data at different spatial resolutions together with a stochastic cloud model would considerably improve the quality of the radiation fields.

The impact that homogeneous clouds and the 1-D RT theory have on retrieval and model products have been extensively investigated in the last years. A wide and comprehen- sive insight into the topic can be found in Marshak and Davis (2005). A more recent literature survey on atmospheric 3-D RT over the last 5 decades is presented in Davis and Marshak (2010). The authors discussed the biases caused by 3-D effects, outlined some mitigation strategies and made suggestions on how 3-D RT itself can be exploited.

Some authors have proposed parameterisations in order to take into account 3-D RT effects in 1-D RT models by defining effective properties of the medium (e.g., Cahalan, 1994; Cairns et al., 2000). Another approach for dealing with cloud variability, but still using the efficient 1-D RT models, is the so-called Independent Pixel/Column Approximation (IPA/ICA). Here, the problem is reduced to a set of 1-D calculations in the columns of a medium with resolved variability (e.g., Cahalan et al., 1994). The domain-averaged radiation fields are computed by averaging the 1-D RT outcomes.

The radiation fields in an inhomogeneous medium calculated as domain averages of IPA calculations differ from those calculated in a plane parallel homogeneous (PPH) medium where the optical properties have been averaged previously. An important reason for this difference is that the radiation fields (e.g., reflectivity and transmissivity) have a nonlinear dependency on the optical depth. To be more specific, the PPH reflectivities (transmissivities) systematically overestimate (underestimate) the IPA/ICA ones. This is a direct consequence of concave (reflectivity) and convex (transmissivity) functions as stated by Jensen's inequality (Jensen, 1906). This bias has been studied under simulated and observed heterogeneous cloudy conditions (Cahalan et al., 1994; Barker, 1996; Oreopoulos and Davies, 1998).

Although the ICA/IPA technique greatly improves the calculation of the radiative transfer by explicitly dealing with cloud variability, it does not account for horizontal radiation transport between atmospheric columns. This horizontal transport is known to be behind some important radiative processes, such as photon channelling, cloud side illumination or cloud side leakage. These effects can lead to the smoothing and/or sharpening of remotely sensed cloud properties as well as biased domain-averaged flux densities. The IPA/ICA bias is defined as the difference between IPA/ICA and fully 3-D calculations. Unlike the PPH bias, the ICA/IPA may both over- or underestimates the 3-D RT results depending on the specific configurations.

O'Hirok and Gautier (1998) investigated different contributions to the IPA/ICA bias by separating the 3-D effects due to cloud morphology and the vertical and horizontal distributions of water vapour, optical thickness and effective radius. They found that IPA/ICA overestimates upwelling irradiance and that cloud morphology is the most important factor for the 3-D effects. Giuseppe and Tompkins (2003) investigated the PPH and IPA/ICA bias with respect to geometrical scales of cloud organization. For this goal, Giuseppe and Tompkins made use of a thermodynamically consistent fractal cloud generator that could control the variability at different scales. 
Authors pointed out that ICA calculations give accurate estimates of reflection, transmission and absorption for overcast clouds. For broken clouds and for scales of $2 \mathrm{~km}$ or less, however, IPA/ICA estimates of the radiation fields could differ by a $5 \%$ from the 3-D RT results. Further, they showed that the solar illumination angle had a severe influence on the estimates, causing IPA/ICA to overestimate reflection for low sun positions and to underestimate reflection for high sun positions.

Varnai and Davies (1999) developed a theoretical framework for studying the processes through which cloud inhomogeneities influence solar radiation. Authors proposed to divide the cloud heterogeneity effects into two main components: the one-dimensional heterogeneity effect and the horizontal transport effect. The first component relates to the sampling of the optical properties in the model. They proposed a new one-dimensional approach called the Tilted Independent Pixel Approximation (TIPA). This approach is based on the IPA/ICA technique, but it samples the cloud properties along a slant column oriented in the direction of the sun. The effects due to the horizontal transport are further divided in four independent mechanisms: upward and downward trapping, and upward and downward escape. These effects occur when photons attempt to move to a thicker or a thinner cloud region, respectively. The authors showed that heterogeneity effects caused by variations in cloud-top height can be comparable to the horizontal transport effect.

Hinkelman et al. (2007) used the framework defined by Varnai and Davies (1999) to study the effect of the anisotropy of cumulus clouds on domain-averaged solar fluxes and heating rates. The cloud cases were obtained from a stochastic cloud generator based on cloud scenes from a large eddy simulation (LES) model. Particularly, they focused on the effect of the tilt and the stretching of the cloud fields.

Marshak et al. (1998) proposed the so-called Nonlocal IPA (NIPA), which incorporates 3-D smoothing effects to the IPA/ICA solution by means of a convolution with an approximate RT Green function. A similar approach was followed by Kniffka and Trautmann (2011) when calculating actinic flux densities. In this case, the 3-D smoothing effects were taken into account by convolution with a Gaussian kernel whose variance is based on the spreading of a light beam through an optically turbid medium.

Zinner and Mayer (2006) simulated the 3-D radiances in a high-resolved marine stratocumulus composed from remotesensed cloud properties in combination with an adiabatic cloud model. Additionally, they also simulated the radiances at a reduced resolution, as it would be collected by spaceborne sensors. After applying 1-D remote-sensing algorithms, they compared the retrieved optical depths with the known ones finding typical underestimations of over $20 \%$.

In many of the studies cited above fractal cloud generators were used to produce the cloud cases for the variability studies. Most of the available cloud generators produce fields having a structure that follows a perfect power law in the wavenumber. However, there are features in real clouds that are not scale invariant - e.g., cloud street, föhn clouds, etc. - affecting the power spectrum. The downscaling algorithm used for the current study (Venema et al., 2010) is based on a stochastic cloud generator that can used measured power spectra including, e.g., waves and scale breaks (Venema et al., 2006).

In the last years, the generation of cloud fields with realistic small-scale variability has received great attention from the atmospheric 3-D RT community. Schutgens and Roebeling (2009) studied the influence that cloud inhomogeneity has on the cloud properties retrieved with different sensors, in particular, when data from one specific retrieval are taken as the reference for the others in validation efforts. Schutgens and Roebeling generated liquid water path variation at smaller scale lengths by considering clouds as simple fractals.

Bugliaro et al. (2011) presented a theoretical case study for the Spinning Enhanced Visible and Infrared Imager (SEVIRI). The authors validated two cloud property retrievals by simulating radiances as they would be measured by SEVIRI in simulated cloud fields. For this purpose, the microphysical properties as given by the COSMO-EU model were statistically downscaled to match the SEVIRI resolution. Montopoli et al. (2012) also introduced a new downscaling scheme, but in this case for increasing the spatial resolution of water vapour observations.

The present paper aims at characterising cloud heterogeneity effects on radiances, namely: The errors due to unresolved variability (the so-called plane parallel homogeneous, $\mathrm{PPH}$, bias) and the errors due to the neglect of transversal photon displacements (independent pixel approximation, IPA, bias). Firstly, 3-D radiative transfer simulations of nadir reflected radiances in the presence of realistic inhomogeneous cloud fields sampled at different spatial resolutions are going to be performed and compared. Secondly, the spectral flux densities integrated over the whole solar range will be computed for a diurnal cycle of a developing cumulus field at different resolutions and using different RT solvers. We will show that the generation of subscale variability from the available cloud properties at a coarse resolution by means of a stochastic cloud model (Venema et al., 2010) greatly reduces the biases in the radiative transfer.

In Sect. 2, we present the Monte Carlo Radiative Transfer (MoCaRT) model which was used to carry out all radiative transfer calculations throughout this paper. For validation, a comparison of MoCaRT with the I3RC-project "consensus" results is included in Sect. 2. The cloud fields used in this paper are presented in Sect. 3. In Sect. 4, we describe the methodology followed to study the effect of the missing variability on the radiative transfer. The results of the study are given in Sect. 5. In Sect. 6, we summarise the paper and draw some conclusions. 


\section{MoCaRT - Monte Carlo Radiative Transfer model}

The Monte Carlo Radiative Transfer model (MoCaRT) is a flexible code designed to address various problems in atmospheric radiative transfer applications.

Its modular structure facilitates the software management and development, since the single parts of the code can be easily reused for new tasks. Furthermore, the user interface is completely separated from the software, so that users do not have to know how the code is actually organised in order to carry out simulations.

MoCaRT has two main components implemented: the optical component and the radiation one. The optical block accounts for the calculation of optical properties from given atmospheric conditions of pressure, temperature, molecular abundances and/or cloud and aerosol microphysics. The surface albedo can be selected for different land compositions (Henderson-Sellers and Wilson, 1983). The solar irradiation can be calculated as the blackbody at Sun's temperature or integrating solar measured or model spectra (Kurucz, 1995). The radiation block accounts for the radiative transfer through the optically active medium defined in the optical part.

MoCaRT offers the possibility of calculating the RT monochromatically, in narrow spectral intervals, or broadband. For monochromatic calculations, MoCaRT computes the absorption coefficients via line-by-line from the HITRAN dataset, where line parameters for the main atmospheric coefficients are listed. In case of narrow intervals, effective absorption coefficients based on the mean interval transmittance are calculated. On request, a correlated $k$-distribution (CKD) approach for an arbitrary interval can be constructed. Broadband computations are performed via the CKD proposed by Fu and Liou (1992) adapted from SHDOM (Evans, 1998).

The optical properties of clouds and aerosols can be calculated in MoCaRT by means of Mie scattering computations. The Mie theory is a general description of the interaction of radiation with spherical particles ${ }^{1}$. The wavelength of the incoming radiation and the particle size and composition (refractive index) are required to compute the scattering, absorption and extinction coefficient, as well as the scattering phase function. Bulk optical properties can be obtained by convolving the properties of individual particles with particle size distributions. Depending on the type of simulation, the particle optical properties are spectrally averaged accordingly. Optionally, efficient parameterisations (but less accurate) can be used for clouds (Slingo, 1989; Stephens, 1994), and aerosols (Shettle and Fenn, 1979; Hess et al., 1998).

MoCaRT can choose between several RT solvers. From the point-of-view of how the variability is taken into account,

\footnotetext{
${ }^{1}$ Note that ice crystals and many aerosol particles are not sufficiently spherical and the optical properties calculated by the Mie scattering theory may be inaccurate.
}

four RT solvers are available. In the plane parallel homogeneous approximation (PPHA), all optical properties are averaged within vertical layers, whereas in the cloudy plane parallel homogeneous approximation (CPPHA), only cloudy optical properties are averaged within vertical layers and the radiation fields are computed combining the cloudy and the clear sky contributions using the cloud fraction $\left(C_{\mathrm{f}}\right)$ as the weight of the cloudy contribution and $\left(1-C_{\mathrm{f}}\right)$ as the weight of the clear sky one. The independent pixel/column approximation (IPA/ICA) (Cahalan et al., 1994) resolves the variability of the optical fields, but the RT is calculated onedimensionally in each atmospheric grid columns. A similar technique that considers independent columns along the solar illumination direction, the so-called tilted independent pixel approximation (Varnai and Davies, 1999) is also implemented. At last, the fully three-dimensional (3-D) solution, where the optical properties are spatially resolved and the transversal photon transport is allowed, is also available.

In order to reach a fast and accurate convergence, several variance reduction and acceleration techniques have been implemented in MoCaRT. Here, we will only describe briefly the diverse implemented techniques.

Usually, photon tracing, i.e., the randomly generation of photon trajectories, is the part of the code that consumes most of the computing time in Monte Carlo RT codes and Mo$\mathrm{CaRT}$ is not an exception. One-dimensional photon tracing algorithms are faster than their three-dimensional counterparts, since they do not have to account for photon horizontal location. Making use of this fact, it is possible to speed up the photon tracing process by considering a 3-D inhomogeneous atmosphere as if it were one-dimensional. This goal is achieved by considering the maximum extinction coefficient values within vertical layers, $k_{\mathrm{ext}}^{\max }(z)$, and introducing a virtual interaction event that left photons unaltered. Assigning the probability weight of $k_{\mathrm{ext}}(x, y, z) / k_{\mathrm{ext}}^{\max }(z)$ to the "maximum extinction" event and $\left(1-k_{\mathrm{ext}}(x, y, z) / k_{\mathrm{ext}}^{\max }(z)\right)$ to the virtual scattering event, the photon tracing is unbiased. The method was described first by Marchuk et al. (1980). They considered the maximum values of the whole medium and called it maximum cross-section method. Since we apply the method for a layered medium, it can be called the stratified maximum cross-section method. This method works well when the maxima are not much larger than the extinction coefficients within layers. Special care has to be taken in case of different phase functions in the medium. We used this method for the flux density simulations presented in Sect. 4.2. In case of radiances, slower but more robust 3-D (or 2-D) tracing algorithms are used.

Several variance reduction methods are related to the manner that the photon-matter interactions are described in the model. The most intuitive method is to describe the history of single photons and their interaction with the medium as they behave in nature: they have constant energy, change direction after scattering events and disappear whenever an absorption event takes place. We refer to this method as "crude Monte 
Carlo". Another method is the so-called "weighted scattering". In this case, the energy of the photons is reduced after each interaction according to the ratio of scattering to extinction, and absorption never occurs. This method can be seen as the representation of a bundle of photons moving in the same direction through the atmosphere, where the energy reduction can be interpreted as the loss of a given number photons due to absorption. A third method considers the interaction of photons with a pure-scattering atmosphere and their energy is continuously attenuated while moving through the atmosphere according to the Beer's law. Note that during the energy attenuation process only the absorption optical thickness is considered. We will refer to this method as "continuous absorption". All these methods are implemented in Mo$\mathrm{CaRT}$ and the appropriateness of one or the other depends on the medium properties and the application.

The estimation of the radiation fields of interest can be performed in situ at place of a virtual detector or summing up contributions of the photons when traversing the atmosphere. In the first case, photon "scoring", i.e., contribution to radiance or flux, is only considered if photons physically reach the detector. This method is appropriate for the computation of fluxes, but not for radiances. In the latter case, the local estimate method (Marchuk et al., 1980) is more suitable. In this method, the contribution is calculated at each interaction point by scoring according to the probabilities of photons reaching the detector, and this allows for a faster convergence.

Making use of the reciprocity theorem, the radiative transfer can be simulated by solving the adjoint equation (backward Monte Carlo method). In this case, "inverse" photons are traced from the detector and the local contributions are summed up according to the probabilities of the photons to reach the source. This method is especially convenient for parallel beam illumination, as in case of solar irradiation. The radiance simulations presented in this paper are computed by means of backward Monte Carlo.

The scattering phase function can take very different shapes. Molecular scattering produces smooth phase functions, whereas the scattering by large particles is described by sharp forward-peaked phase functions. In the latter case, the convergence to the solution is slowed down and a higher number of realisations (photons) is needed. In order to accelerate convergence, the regionalisation method of the local estimate technique presented in Barker et al. (2003) is implemented. The truncated radiance contributions - up to a given "tunable" threshold - are summed up as usual, and the contributions that exceed this threshold are stored in a scene "radiance surplus bucket". After the computation, the bucket is decanted over the scene such that regions with higher truncated radiances linearly become more portion of the radiance bucket. This method delivers smoother radiation fields using less number of photons. The scene mean value is unbiased, but local biases may occur depending on the contribution threshold. The only difference to the Barker et al. regionali- sation method is that our radiance contributions include the single scattering albedo at the locations of each scattering event (weighted scattering method).

\section{Validation}

In order to validate the MoCaRT model, this section presents a comparison of MoCaRT with the consensus results of the Intercomparison of Three-Dimensional Radiation Codes (I3RC) project (Cahalan et al., 2005). All MoCaRT results presented here were obtained using the weighted scattering method previously described.

The I3RC project was conceived with the goal of comparing a wide variety of three-dimensional radiative transfer models applied to Earth's atmosphere. During the phase I of the project, several baselines for 3-D radiative transfer computations through inhomogeneous clouds were defined. These computations are based upon three cloud cases: a 1-D academic "step" cloud field, a 2-D field derived from radar and microwave observations of the Atmospheric Radiation Measurement (ARM) programme, and a 3-D field derived from radiances measured by the Landsat 5 Thematic Mapper instrument. We performed the simulations suggested in phase I of I3RC and some selected results of flux densities and radiances are presented next.

\section{Intercomparison of Three-dimensional Radiation Codes (I3RC)}

The first case of the phase I of the I3RC project is a one-layer "step cloud" consisting of 32 pixels along the horizontal dimension. The first 16 pixels have an optical depth of 2 and the remaining ones of 18 , resulting in a domain-average optical depth of 10. The horizontal extension of the cloud is of $0.5 \mathrm{~km}$, whereas the vertical one is of $0.25 \mathrm{~km}$ everywhere, i.e., a flat cloud. This case allows for testing the model behaviour around a region with large optical depth gradient, i.e., the sharp transition from low to high cloud optical depth (see http://i3rc.gsfc.nasa.gov/input/step_cloud/index.html for detail).

The second case consists of a 2-D cloud field based on extinction retrievals from the combined measurements of the Millimeter Cloud Radar (MMCR) and the Microwave Radiometer (MWR) at the Atmospheric Radiation Measurement (ARM) CART site in Lamont, Oklahoma. The field consists of 640 columns along the horizontal axis. The horizontal width of the columns is of $50 \mathrm{~m}$ according to the measurement integration time (10 s) and the observed wind speed $\left(\sim 5 \mathrm{~m} \mathrm{~s}^{-1}\right)$. Vertically, the field is resolved into 54 layers of $45 \mathrm{~m}$ thick each and extends from about $0.6 \mathrm{~km}$ to $2.43 \mathrm{~km}$ above the Earth's surface (see http://i3rc.gsfc.nasa.gov/input/ MMCR/high_res/020898/index.html for detail).

The third case is based on a two-dimensional (2-D) cloud field extracted from a Landsat- 4 scene. The optical depth field consists of $128 \times 128$ vertically homogeneous 

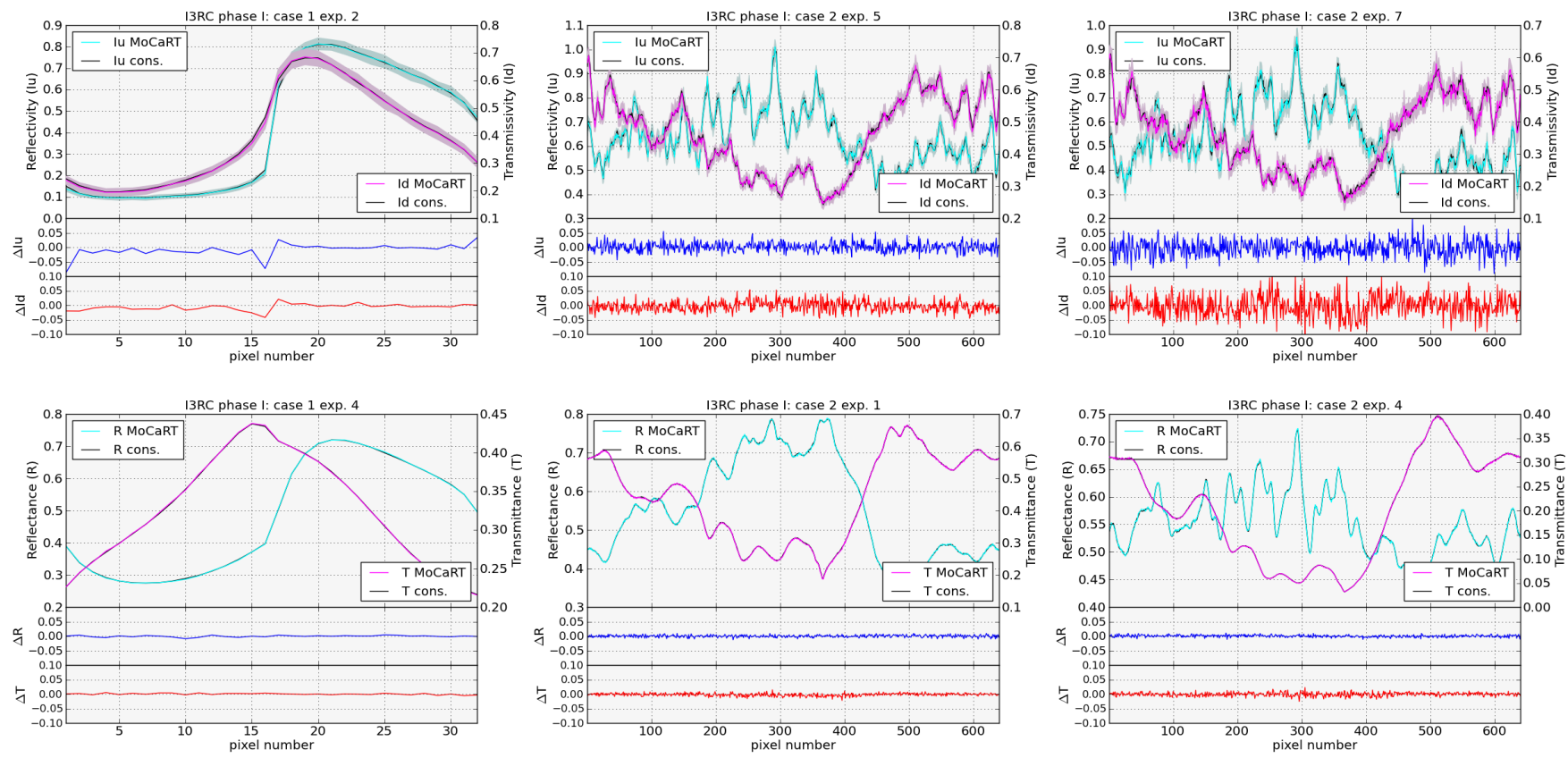

Fig. 1. I3RC project phase I. Case 1 represents a step cloud of mean optical thickness of 10. Case 2 represents a measured 2-D cloud field. MoCaRT results are compared to the consensus results of the participants in the I3RC project. The upper row presents radiance results (reflectivities and transmissivities), whereas the lower row presents flux densities (reflectances and transmittances). The left column illustrates results of the case 1 and the central and right column of case 2. The central (lower) panel of all subplots illustrates the reflectivity/reflectance (transmissivity/transmittance) relative difference. The local discrepancies are few percent for all cases, except for the transmissivity of experiment 7 of case 2, where the discrepancies exceed $5 \%$ in some regions. All cases are well within the error bars as illustrated in upper panels.

horizontal columns. The column width is $30 \mathrm{~m}$ in both horizontal directions. In order to build up a three-dimensional (3-D) spatial cloud field, a constant cloud bottom at $0.2 \mathrm{~km}$ was considered and cloud top heights were determined from a separated field of geometrical thicknesses. The cloud fraction is 0.884 and the domain-average cloud optical depth (i.e., considering only the cloudy regions) is 11.4 (see http: //i3rc.gsfc.nasa.gov/input/Landsat/index.html for detail).

Many institutions took part in the I3RC project contributing with different models. Combining the results of the best models, the so-called "consensus" results have been created and made available at the website of the I3RC project. We compare here the MoCaRT with the I3RC consensus results for validation.

Figure 1 shows a comparison of RT results for cases 1 and 2 . Both clouds were considered to extend to infinity along the horizontal y-direction. No atmospheric effects were considered. The surface was black, i.e., the surface albedo was set to zero, except for the experiment 5 of case 2 (middle upper subplot) where it was set to 0.4. The HenyeyGreenstein scattering phase function with an asymmetry parameter of 0.85 was assumed throughout the cloud for all cases except for the experiment 7 of case 2 (right upper subplot), where the $\mathrm{C} 1$ scattering phase function (Deirmendjian, 1964) was used. The single scattering albedo, i.e., the ratio of scattering to extinction coefficient, was set to the unity (pure scattering) for all cases except for the experiment 4 of cases 1 and 2 (left and right lower subplots). The sun was overhead for both cases of the central column and oblique with a solar zenith angle of $60^{\circ}$ for the cases shown in the left and right columns. The upper row shows radiances and the lower row presents flux densities. The transmissivities and reflectivities were calculated for zenith and nadir view directions. The local discrepancies in reflectivities and transmissivities are few percent for all cases, except for the transmissivity of the experiment 7 of case 2 , where the discrepancies exceed $5 \%$ in some regions. In case 1, there are discrepancies in the transition regions from low to high optical depth and vice versa. The reason for these discrepancies is that we calculated the radiances at the centre of the pixels and not averaged over the whole pixel. The relative differences in case of reflectances and transmittances are lower than $1 \%$. All cases are well within the error bars as illustrated in the upper panels. The domain averaged quantities agree better than $1 \%$.

Figure 2 shows the comparison results relative to case 3 . Since in this case the extinction field is three-dimensional, it is necessary to compare the two-dimensional radiative quantities in separate graphs. The upper subplots present reflectivity results and the lower ones absorptance. The surface albedo was set to zero and the Henyey-Greenstein scattering 

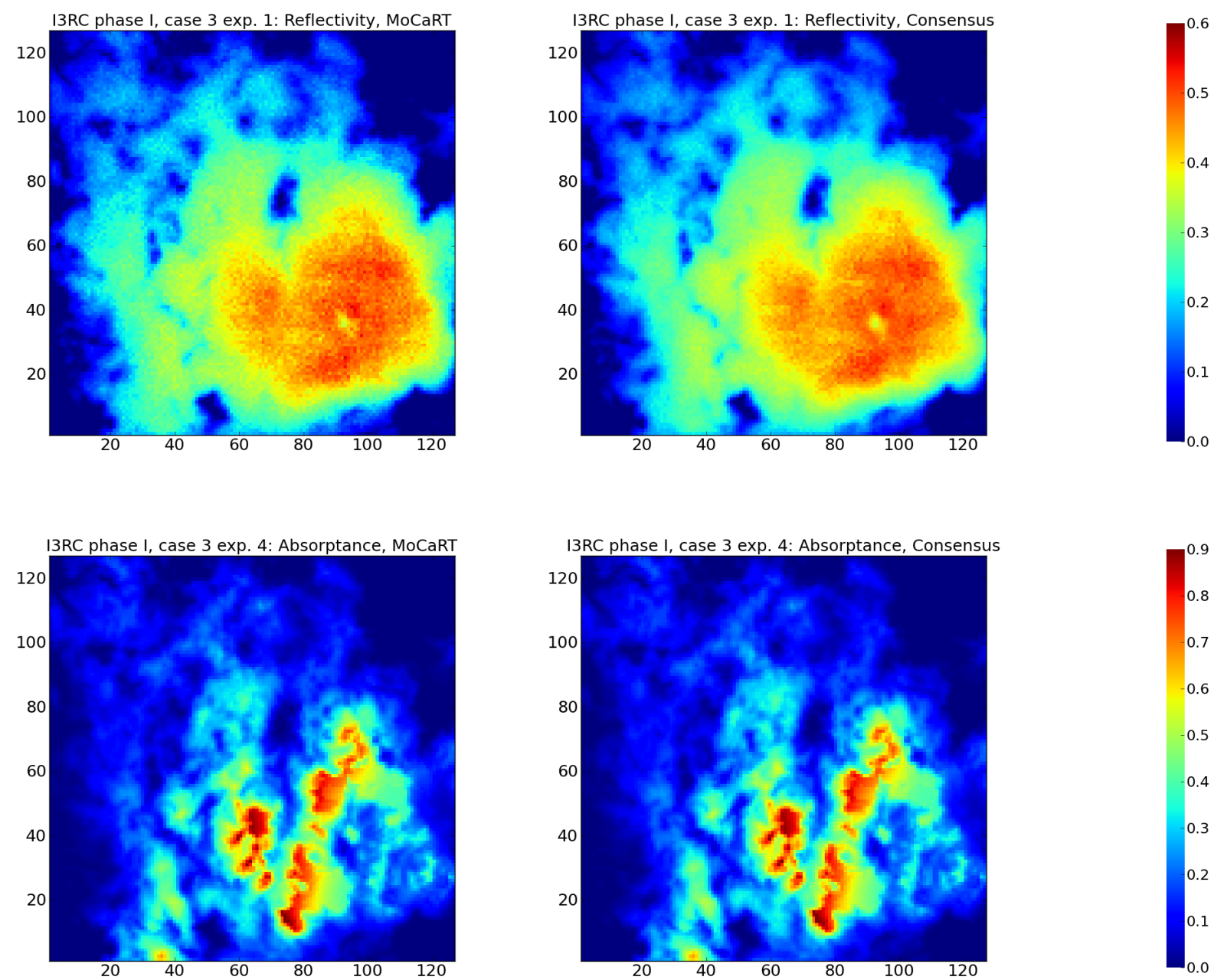

Fig. 2. I3RC project phase I. Case 3 represents a Landsat cloud. MoCaRT results are compared to the consensus results of the participants in I3RC project. The left column shows the MoCaRT and the right column, the consensus results. The top row illustrates the reflectivity fields and the bottom one the absorptance. Differences are well within the Monte Carlo noise.

phase function with an asymmetry parameter of 0.85 was assumed throughout the cloud for both experiments, 1 and 4. The single scattering albedo was 1 and 0.99 , respectively. The solar zenith angle was set to $0^{\circ}$ (upper row) and $60^{\circ}$ (lower row). Although the extinction field is highly variable and the solar illumination is not perpendicular, the figures provided by both methods are almost identical. The mean, maximal and minimal values agree better than $0.1 \%$, which is clearly better than the required accuracy in the I3RC project.

\section{Cloud fields}

In this section, we present the three-dimensional inhomogeneous cloud fields that have been used in this paper as a framework for the study of the cloud variability effect on the radiative transfer.
By means of current measurement techniques, it is only possible to capture either a two-dimensional (2-D) crosssectional or an incomplete three-dimensional (3-D) view of clouds. The most suitable option for overcoming this lack of information and obtaining a cloud in a fully 3-D fashion is to generate the missing variability by means of cloud dynamical and/or stochastic models. Hence, all 3-D clouds presented in this paper are synthetic fields.

In order to produce "realistic" 3-D clouds from observations, one can use dynamical models to simulate the formation and evolution of clouds at a fine temporal and spatial scale. In such cases, the gap in (measured) cloud properties and detailed cloud structure is filled by modelling the physical processes that take place within the embedding atmospheric scenario. The link to reality is achieved by feeding the models with the actual atmospheric conditions, mainly profiles of temperature, pressure, moisture and wind velocity. 

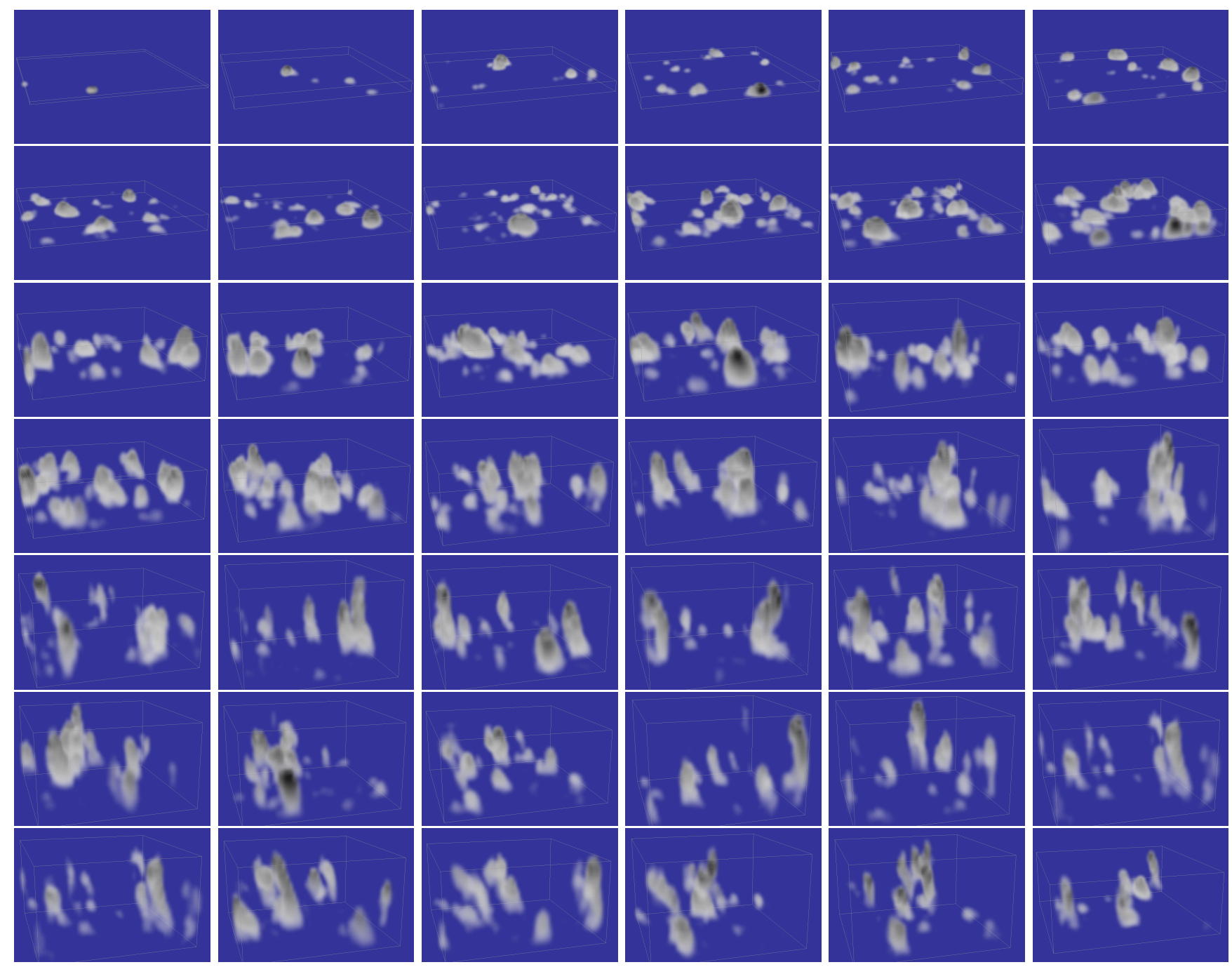

Fig. 3. Large eddy simulation of the diurnal cycle of shallow cumulus convection over SGP site of the ARM program (Brown et al., 2002). The snapshots represent the liquid water content fields (light/dark grey for lowest/highest LWC) sampled each 20 min from 08:40 UTC until 22:20 UTC.

In this study, a dataset of cloud properties of a modelled diurnal cycle of a shallow cumulus over land (Brown et al., 2002) was used. They performed large eddy simulations (LES) initialised with observations carried out on 21 June 1997 at the Southern Great Plains (SGP) site of the Atmospheric Radiation Measurement (ARM) program. The SGP Central Facility is located at Ponca City, OK (latitude: $36^{\circ} 36^{\prime} 18^{\prime \prime}$, longitude: $\left.97^{\circ} 29^{\prime} 6^{\prime \prime}\right)$. The model supplied 51 highly resolved scenes of the evolving cloud every $20 \mathrm{~min}$ from 07:00 UTC until 24:00 UTC. The cloudy scenes are composed of 64 by 64 cells in the horizontal $\mathrm{x}$ - and $\mathrm{y}$-axes and 122 in the vertical dimension (from which only those between 1160 and $3040 \mathrm{~m}$ were used in this study). The horizontal resolution is $100 \mathrm{~m}$ and the vertical $40 \mathrm{~m}$. Figure 3 illustrates the LES cumulus cloud liquid water content (LWC) development. Note that the graphics are 3-D plots from a slant perspective. The plotted sequence starts at 08:40 UTC (top left scene) and finishes at 22:20 UTC (bottom right). The initial and final scenes have been omitted for illustration convenience. Dark areas represent regions with high LWC and bright areas regions with low LWC. The background has been colored blue to emphasise the cloud structure. The purpose of this figure is to stress the complex shapes that clouds can take rather than provide concise information on LWC absolute values. Note that in order to facilitate the visualisation of the sequence as a whole, the colourbar has been left out. In the morning, the SGP site was under an inversion situation that led to a stable shallow boundary layer (until third scene of the second row). Some time after midday, the net surface heat became positive, the PBL warmed up and shallow cumulus convection started (from fourth scene of the second row on). 
These highly variable three-dimensional clouds were used in this paper as reference for the variability studies presented in the next section.

\section{Study - impact of missing variability}

The accuracy of the radiative transfer solution in an inhomogeneous medium depends on the spatial resolution at which the optical properties are defined. In applications such as climate modelling, weather prediction or remote sensing of atmospheric and surface variables, the definition of variability down to a fine resolution is typically not possible or suitable due to computing time limitations. If the optical properties are only defined up to a coarse resolution, a bias is introduced to the simulated radiation fields. In this section, we present two studies about the effect of the missing variability on the radiative transfer: one for radiances and the other for flux densities. Two main goals are pursued in the studies: quantifying the biases, on the one hand, and proposing a method for correcting them, on the other.

In order to achieve the first goal, the cumulus clouds at high horizontal resolution presented in the previous section are taken as reference for the radiation studies. A second cloud dataset with lower resolution is produced from the reference one. The coarse cloud fields were computed by averaging $16(4 \times 4)$ columns of the original clouds leading to a horizontal resolution of $400 \times 400 \mathrm{~m}$. The vertical resolution was left unchanged at $40 \mathrm{~m}$. Hence, calculating the radiative transfer in this second cloud set with coarser resolution and comparing the results to the original cloud set with finer resolution, we can quantify the biases associated to the neglect of horizontal variability below $400 \mathrm{~m}$.

Venema et al. (2010) developed a downscaling algorithm for cloud fields, that generates high-resolution 3-dimensional cloud fields based on coarse resolution cloud water and cloud fraction fields. This algorithm generates clouds with realistic subscale variability that complements the resolved cloud field and makes radiative transfer computations more accurate. This latter statement can be tested, if we take the coarseresolved $(400 \times 400 \mathrm{~m})$ cloud set as the starting point, generate cloud variability down to a resolution of $100 \times 100 \mathrm{~m}$, and compare the results with the original cloud set, also of $100 \times 100 \mathrm{~m}$ horizontal resolution, from which the coarseresolved cloud set was calculated.

In the next subsections, we describe in detail the two studies for investigating the effects of the missing variability on radiances and flux densities.

\subsection{Effect of missing variability on radiances}

Radiance observations by remote-sensing instruments on board spacecraft and aircraft platforms or on ground-based stations can be used to obtain information about the thermodynamic state of the atmosphere, including the content of the main atmospheric molecules, cloud condensates and aerosols. In general, the signal measured by atmospheric sensors have contributions from a large portion of the atmosphere, where the probability of containing cloud condensates is high. It is common practice in atmospheric composition remote sensing to filter out the cloudy scenes or to neglect cloudiness below a certain threshold (e.g., for cloud fractions below $5 \%$ ). Other algorithms consider the clouds as homogeneous blocks that cover a fraction of the observed scene and the rest of the scene as clear sky. In these cases, cloud variability is suppressed within the cloud fields and the photon transport from the cloudy to the clear sky region is not allowed.

For surface remote-sensing applications, sensors are typically provided with a much higher spatial resolution than their atmospheric counterparts. Cloud masking algorithms are used to filter out cloudy pixels, but in case of thin clouds (e.g., cirrus) or clouds over bright surfaces (e.g., ice or desert areas), these algorithms may fail. In order to retrieve surface properties, atmospheric effects have to be corrected. Surface retrieval algorithms work at high spatial resolution, but the transversal photon transport is not allowed.

These limitations imposed to the radiative transfer solvers introduce biases to the radiation results and, consequently, also biases to the retrieval products.

Here, we present a study on the effect of the spatial resolution on measured reflectivities and propose a method for improving the results. Let us assume that we have defined the cloud properties at a horizontal resolution of $400 \times 400 \mathrm{~m}$. We will refer to these clouds as "coarse" fields. Additionally, by means of the downscaling method presented in Venema et al. (2010), we produced a new cloud set with horizontal variability down to $100 \times 100 \mathrm{~m}$. The downscaling method accepts measured as well as theoretical power spectra of cloud properties and, thus, allows for a realistic description of the variability at the small spatial scales. In this case study, we use the cumulus clouds of Sect. 3 as reference, so that the radiation results can be directly compared to them. Hence, deviation of the reflectivities from the original cloud set will be interpreted as biases and, accordingly, we can test the impact of the spatial resolution as well as the proposed improvement method by generating the missing variability.

Since this study is focused on the cloud spatial variability, we did not consider any atmospheric effect, i.e., the cloud fields were embedded in vacuum, neither molecular absorption or scattering was considered, nor aerosol extinction. Lambertian reflection at the flat surface with an albedo of 0.1 was considered. The cloud scattering properties were calculated by means of the parameterisation proposed by Slingo (1989). The phase function was approximated by the Henyey-Greenstein phase function with the asymmetry parameter calculated from the aforementioned parameterisation. Two solar zenith angles were considered, $0^{\circ}$ and $60^{\circ}$. The reflectivities were calculated for a nadir viewing instrument. The same simulations were repeated for all 49 cloudy 

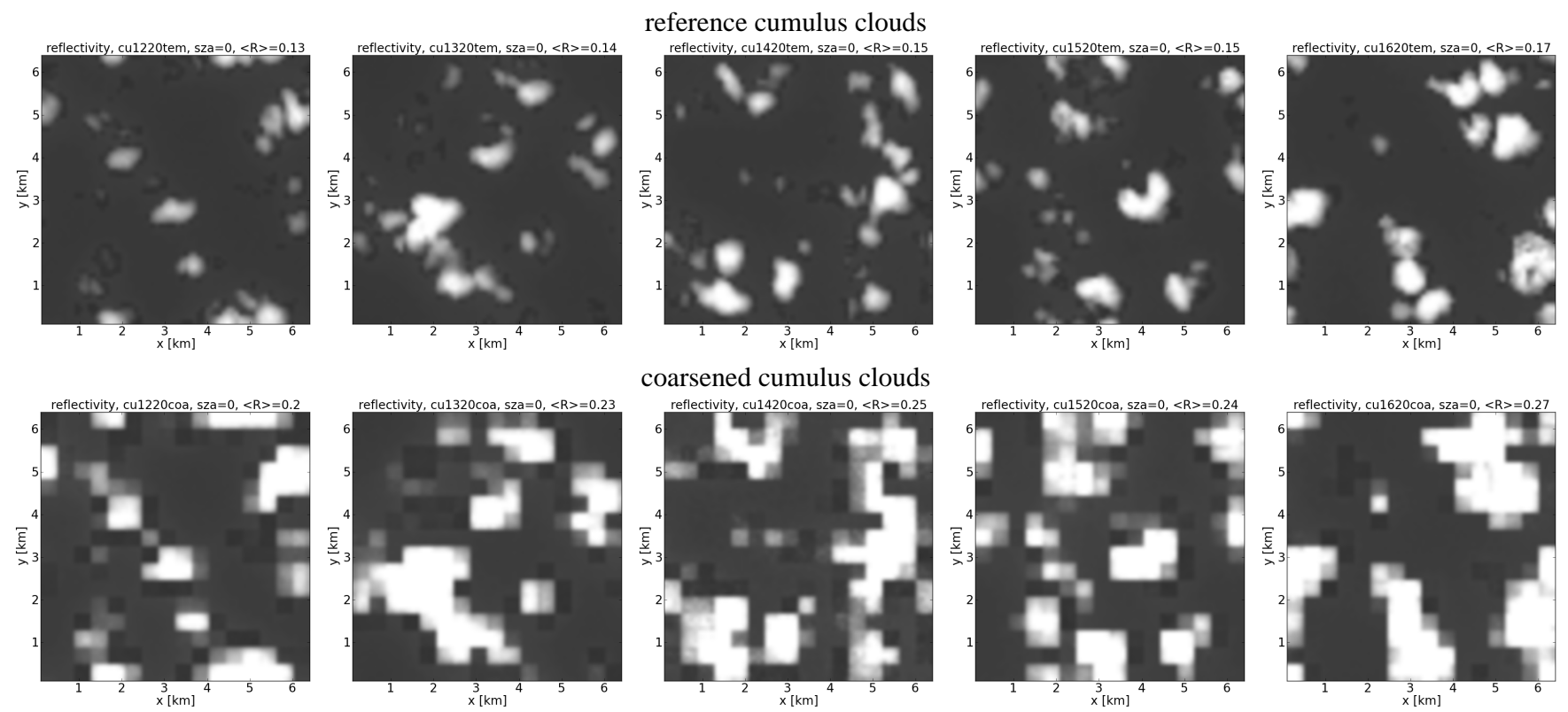

coarsened cumulus clouds
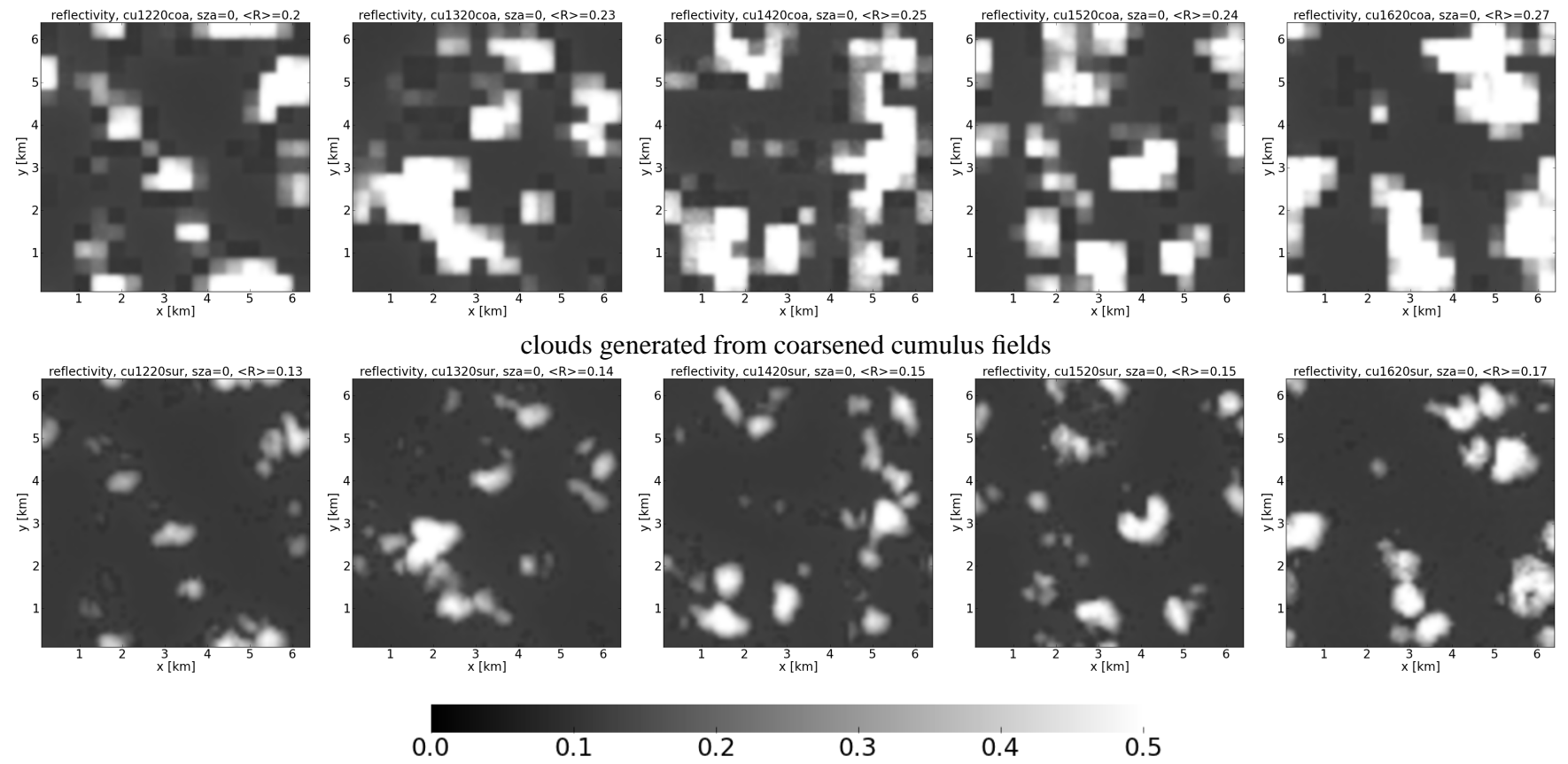

0.2

0.3

0.4

0.5

Fig. 4. Reflectivity fields of some selected scenes of the cumulus diurnal cycle presented in Sect. 3 . The sun is overhead $\left(\mathrm{SZA}=0^{\circ}\right)$ and the observer looks exactly in the nadir direction. The top row corresponds to the LES clouds considered here as the reference (see Fig. 3 ). The middle row represents the coarsened cloud reflectivities. The bottom row illustrates the reflectivities calculated at clouds generated from the coarsened ones by adding the missing sub-scale variability. Notice the similarity of the top and bottom row fields indicating the good performance of the downscaling method.

scenes of all three cloud sets: the coarse, the downscaled and the reference one.

Figures 4 and 5 illustrate reflectivity scenes randomly selected from all cloud cases for solar zenith angles of $0^{\circ}$ and $60^{\circ}$, respectively. The upper row presents the reflectivities computed in the original cloud set which will be considered here as the real cloudy conditions. The reflectivities computed in the coarsened cloud fields are presented in the central row, where the lack of small detail is manifest. The lower row presents the reflectivities in the cloud fields with stochastically generated small-scale variability. One can see that the reflectivities resemble the ones of the original fields, indicating the benefit of calculating the radiative transfer at spatially high-resolved cloud fields. Notice the realistic shadows of the cloud fields on the ground in Fig. 5. In this illustration, the oblique sun is illuminating from the South.
In Sect. 5.1 domain-averaged reflectivities are analysed. These results can be interpreted in terms of the impact on cloud property retrievals. Reflectivity is a function of cloud optical depth (among others). By means of the asymptotic theory for thick atmospheres (see e.g., King, 1987; Nakajima and King, 1990), a straightforward conversion of reflectivities into optical thicknesses can be achieved and the differences found in this study interpreted in terms of retrieved optical thickness. For such a study we refer to, e.g., Zinner and Mayer (2006); Varnai and Marshak (2002).

\subsection{Effect of missing variability on radiative fluxes: a diurnal cycle}

Together with the impact of cloud variability on radiances, we also investigate the effect on flux densities. In particular, 

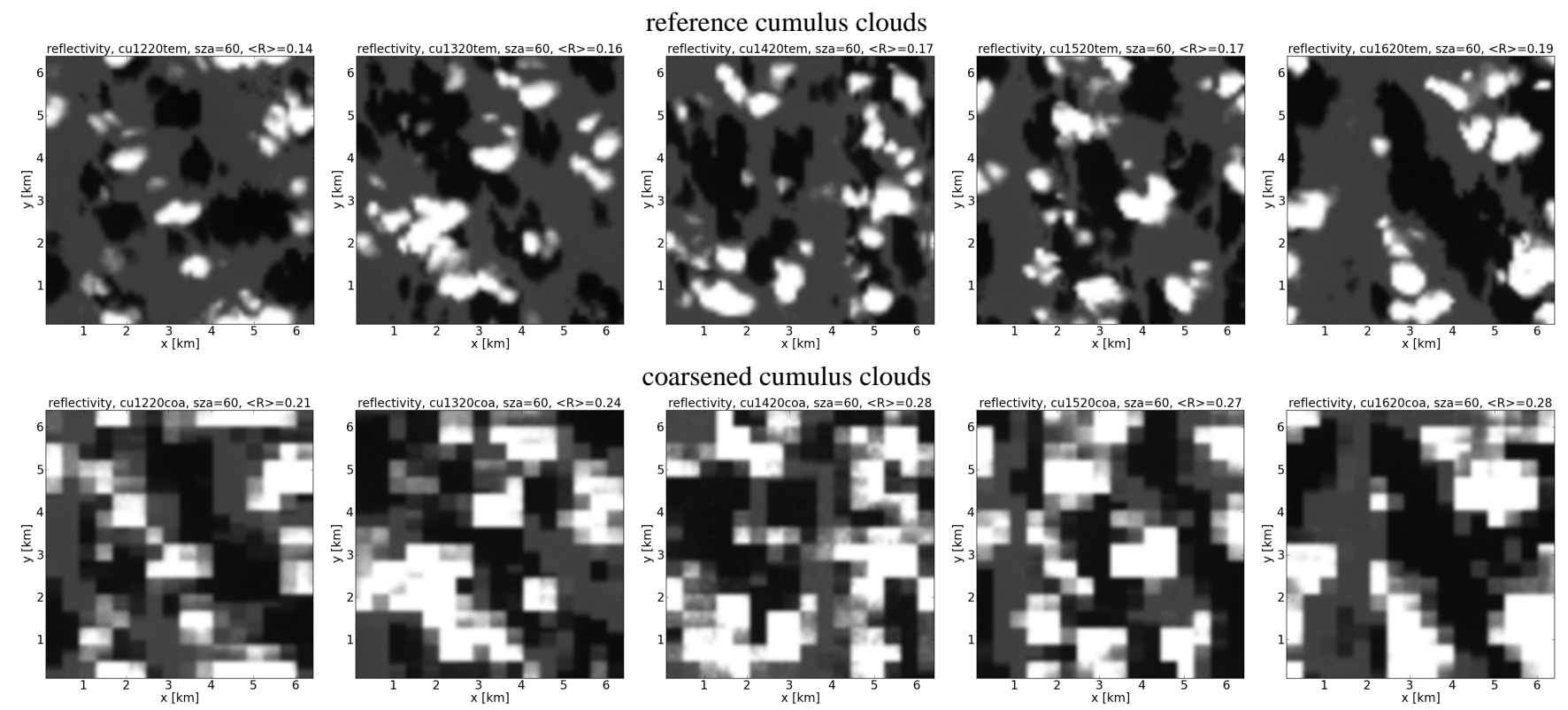

coarsened cumulus clouds
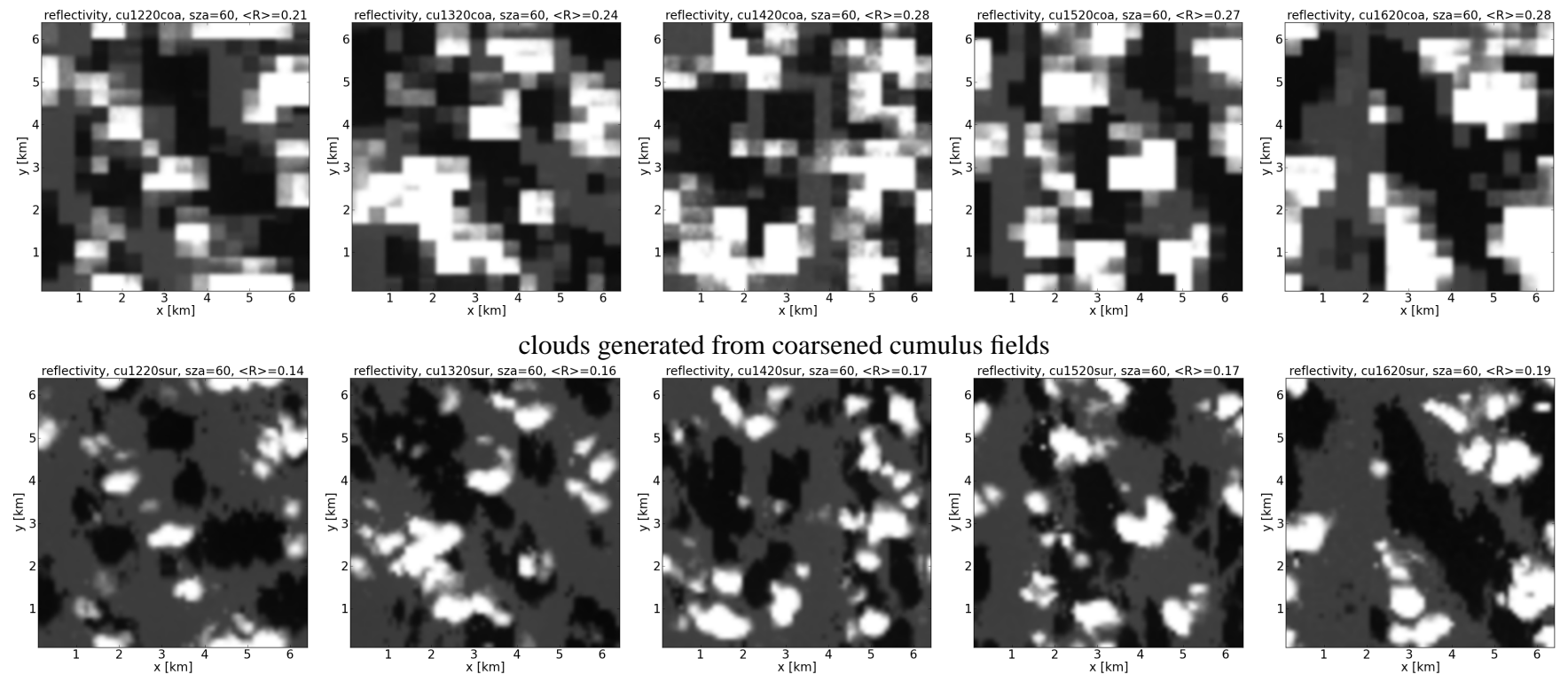

0.0

0.1

0.2

0.3

0.4

0.5

Fig. 5. Reflectivity fields of some selected scenes of the cumulus diurnal cycle presented in Sect. 3 . The sun illuminates the scenes from the south at a zenith angle of $60^{\circ}$. Otherwise, the figure is the same as Fig. 4. Notice the similarity in the top and bottom row fields indicating the convenience of calculating the radiative transfer at spatially high-resolved cloud fields.

we have studied the impact on flux densities integrated over the whole solar region of the spectrum. Thermal radiation was not considered. The designed scenario for this study is presented next.

The diurnal cycle of the convective cumulus presented in Sect. 3 (see Fig. 3) was embedded into a model atmosphere over land. Only the troposphere and the lower stratosphere (top of the atmosphere was set at $30 \mathrm{~km}$ ) were considered. The cloud optical properties were calculated from the microphysical properties by means of the parameterisation proposed by Slingo (1989). The angular distribution of cloud scattering events was described by the Henyey-Greenstein phase function with the asymmetry parameter obtained previously from the mentioned parameterisation. The solar position (zenith and azimuth) was exactly calculated as a function of time and geolocation (see details in Sect. 3). The broadband molecular absorption was taken into account by means of the correlated $k$-distribution (CKD) given by Fu and Liou
(1992). Molecular (Rayleigh) scattering coefficients were calculated by the formula given by Nicolet (1984) and averaged over the broadband intervals of the $k$-distribution. The effect of aerosols was neglected. Lambertian reflection at the surface was considered with a broadband surface albedo corresponding to an agricultural region (Henderson-Sellers and Wilson, 1983).

The RT simulations were performed using the Monte Carlo Radiative Transfer model (MoCaRT), which can provide both, the 3-D exact solution of the RT or an approximate one by employing a variety of 1-D methods.

On the one hand, we carried out fully 3-D calculations over the whole day using the fine resolved cumuli $(100 \mathrm{~m} \times 100 \mathrm{~m} \times 40 \mathrm{~m})$ and considered the results of these simulations as reference ("the truth"). On the other hand, we calculated the RT by means of the independent column approximation (ICA) using coarser clouds, representing the RT 

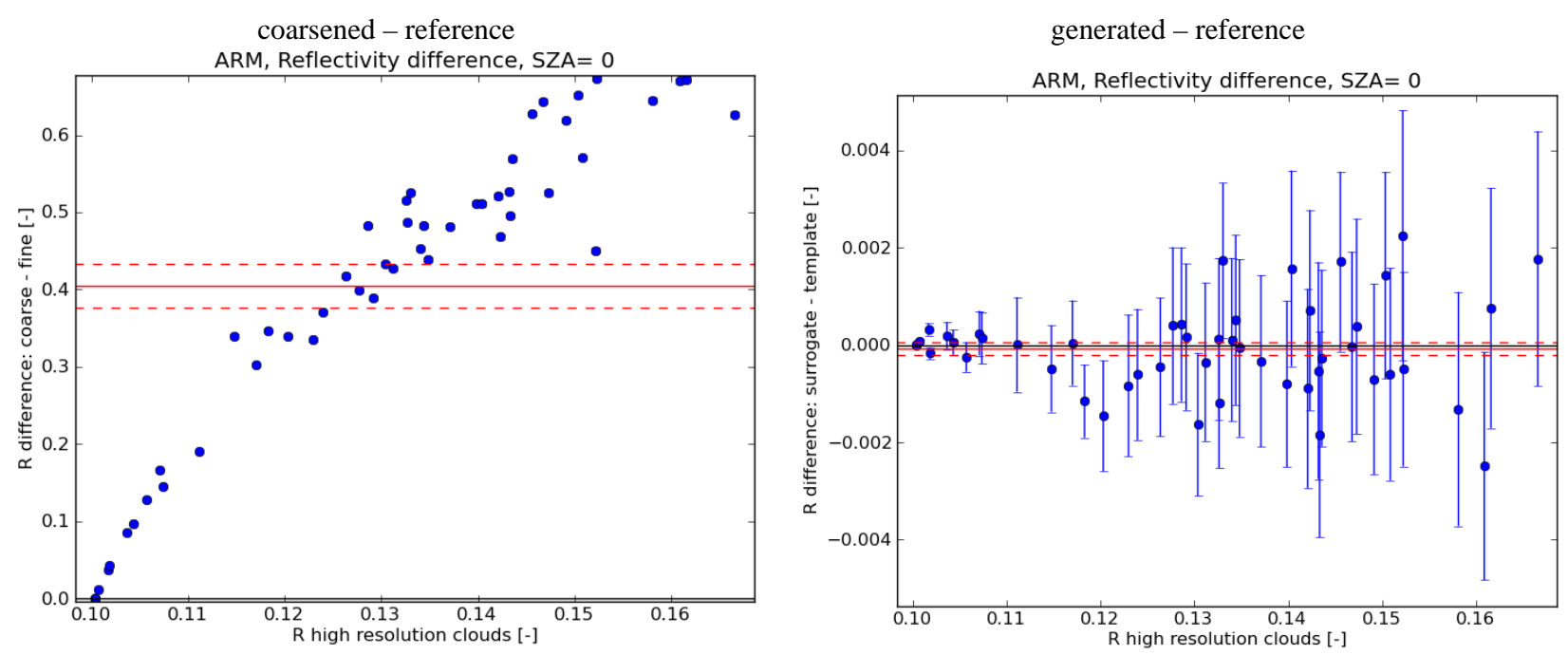

Fig. 6. Differences in domain-averaged reflectivity. The solar zenith angle was set to $0^{\circ}$. The abscissa indicates the mean reflectivity of the reference clouds and the ordinate indicates the relative differences. The blue dots represent the differences within a single cloudy scene and the error bars the corresponding standard deviations. The solid red line represents the mean bias of all cloud fields and the dashed red lines, the mean plus and minus the standard deviation, respectively.

scheme in a cloud resolving model with coarser spatial resolution.

\section{Results}

Domain-averaged results of the studies presented in Sect. 4 are shown here. The results are presented separately: first, we compare the reflectivities of the coarse and the downscaled clouds with the reference ones; secondly, we compare the fluxes of the coarse cumulus clouds with the reference ones during the diurnal cycle.

\subsection{Reflectivity: difference plots}

Figure 6 shows difference plots of domain-averaged reflectivities between the coarse and the reference clouds (left panel) and the downscaled and the reference clouds (right panel) for a solar zenith angle of $0^{\circ}$. The abscissa indicates the mean reflectivity of the reference clouds and the ordinate indicates the relative differences $\Delta R$ defined as:

$\Delta R:=\left(R_{\mathrm{coa}}-R_{\mathrm{ref}}\right) / R_{\mathrm{ref}}$,

where $R_{\text {coa }}$ represents the domain-averaged reflectivity in the coarsened cloud fields and $R_{\text {ref }}$ the domain-averaged reflectivity in the reference cloud fields at the original high spatial resolution. The blue dots represent the differences within a single cloudy scene and the error bars the corresponding standard deviations. The solid red line represents the mean bias of all cloud fields and the dashed red lines, the mean plus and minus the standard deviation, respectively. Fully 3D RT calculations were performed in all cases. Thus, the reflectivity differences cannot be attributed to restrictions in the radiative transfer, but have to be entirely due to the resolution at which the optical properties are defined. The loss of variability leads to an overestimation of the reflectivity in case of the coarse-resolved clouds due to Jensen's inequality (see left panel). Notice the general tendency that the higher the reflectivities (i.e., higher optical depths), the higher the differences between the coarse clouds and the reference ones. The mean bias introduced only by defining the cloud fields at a coarser resolution is about $40 \%$. If cloud variability is generated by means of stochastic methods and added to the coarse clouds, this bias is eliminated (see right panel).

Figure 7 is equivalent to Fig. 6 except that in this case the solar zenith angle was set to $60^{\circ}$. The same conclusions found in Fig. 6 hold for oblique illumination. The mean bias due to the lack of variability is of about $35 \%$. Again, the generation of small-scale cloud variability helps to reduce the mean bias. In this case, the downscaled mean bias is about $1 \%$.

\subsection{Radiative fluxes: a diurnal cycle}

We will study the errors made when calculating onedimensionally the solar radiation fluxes within coarseresolved cloudy atmospheres instead of highly resolving cloud horizontal variability and accounting for the photon horizontal transport.

Figure 8 shows the results of the study. The left panel shows the reflected fluxes at the top of the atmosphere (TOA), the right top panel shows the transmitted fluxes at Earth's surface and the right bottom panel shows the absorbed fluxes through the whole atmosphere. The absolute values of the fluxes depend on the incoming solar radiation 

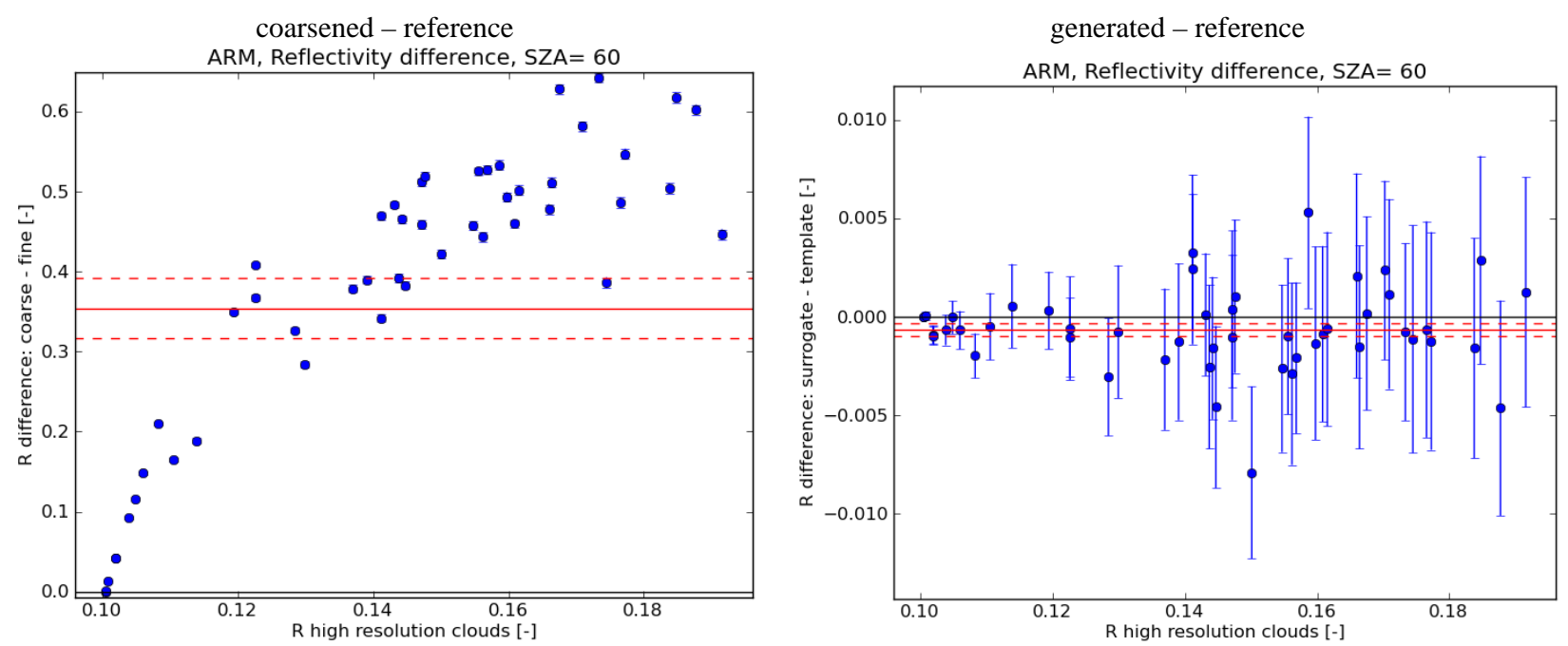

Fig. 7. Same as Fig. 6 but for a solar zenith angle of $60^{\circ}$.

which is a function of the cosine of the solar zenith angle (SZA), which is clearly seen in all three plots. The red line represents the difference between the coarse and the high resolved fluxes:

$\Delta X:=X_{\text {coa }}-X_{\text {ref }}$,

where $X$ stands for reflected, transmitted or absorbed flux. Note that the flux differences have been multiplied by a factor of 10 ( -10 in case of transmitted flux) in the figures in order to include them in the same frame as the absolute values, without losing details.

Two main effects contribute to the differences in the fluxes. First, cloud variability is described at different resolutions and, second, horizontal photon transport is allowed in one case and forbidden in the other. The loss of variability leads to an overestimation (underestimation) of the reflected (transmitted) fluxes in coarsely resolved clouds (ICA-PPA bias). Additionally, ICA simulations neglect the radiative communication between atmospheric columns (3-D-ICA bias). This second bias depends on the spatial distribution of the cloud properties as well as on the illumination geometry and can lead to both, over- and underestimations. For instance, downwelling photons intercepted by cloud sides can undergo scattering events within the clouds, changing to upwelling direction and later contributing to reflection (see, e.g., O'Hirok and Gautier, 1998). This mechanism can be simulated in 3D calculations, but not in the ICA, leading to an underestimation of the ICA reflected flux. The impact will obviously be more important for low sun. However, in our study reflected flux is systematically overestimated in the ICA-coarse simulations and, consequently, this mechanism cannot be the dominant one.

The maximum discrepancies in reflected and transmitted flux occur at 15:00 UTC and 16:20 UTC (2nd and the 6th snapshots of the 4th row in Fig. 3), well after noon. As men- tioned in Sect. 3, shallow cumulus convection started after midday and, at those times, the cumulus cloud had already considerably developed in the vertical. These maximum deviations are then a combination of moderately high incoming solar flux (although less than at noon) and complex cloudy structure.

In our study, the ICA-coarse (green spots) reflected fluxes are larger than their 3-D-fine counterparts (blue spots) over the whole day with maxima as large as $30 \mathrm{~W} \mathrm{~m}^{-2}$. The standard deviation of the mean is shown as error bars: the larger the error bars, the higher the dispersion of the values. As a consequence of horizontal transport, 3-D-fine reflected fluxes at TOA are smoother than the ICA-coarse ones. In case of transmitted fluxes at the ground, 3-D-fine fields show large variability, since the cloud layers are close to the surface.

\section{Conclusions and outlook}

In inhomogeneous cloudy atmospheres, the radiative transfer strongly depends on the horizontal scale at which the microphysical properties of the cloud fields are defined. The neglect of cloud variability introduces biases when simulating the radiation transfer. These biases affect the accuracy of remote-sensing applications and climate and weather prediction models.

Reflectivities are important for remote sensing. We studied the effect that cloud resolution has on reflectivities. We showed that considering the cloud properties at a horizontal resolution of $400 \mathrm{~m}$ by $400 \mathrm{~m}$ introduces a bias of about $40 \%$ for overhead sun and $35 \%$ for a solar zenith angle of $60^{\circ}$ when averaging over 49 cumulus scenes of different cloudiness in comparison to a resolution of $100 \mathrm{~m}$ by $100 \mathrm{~m}$. Additionally, we showed that generating subscale variability by means of stochastic methods and adding this variability to 

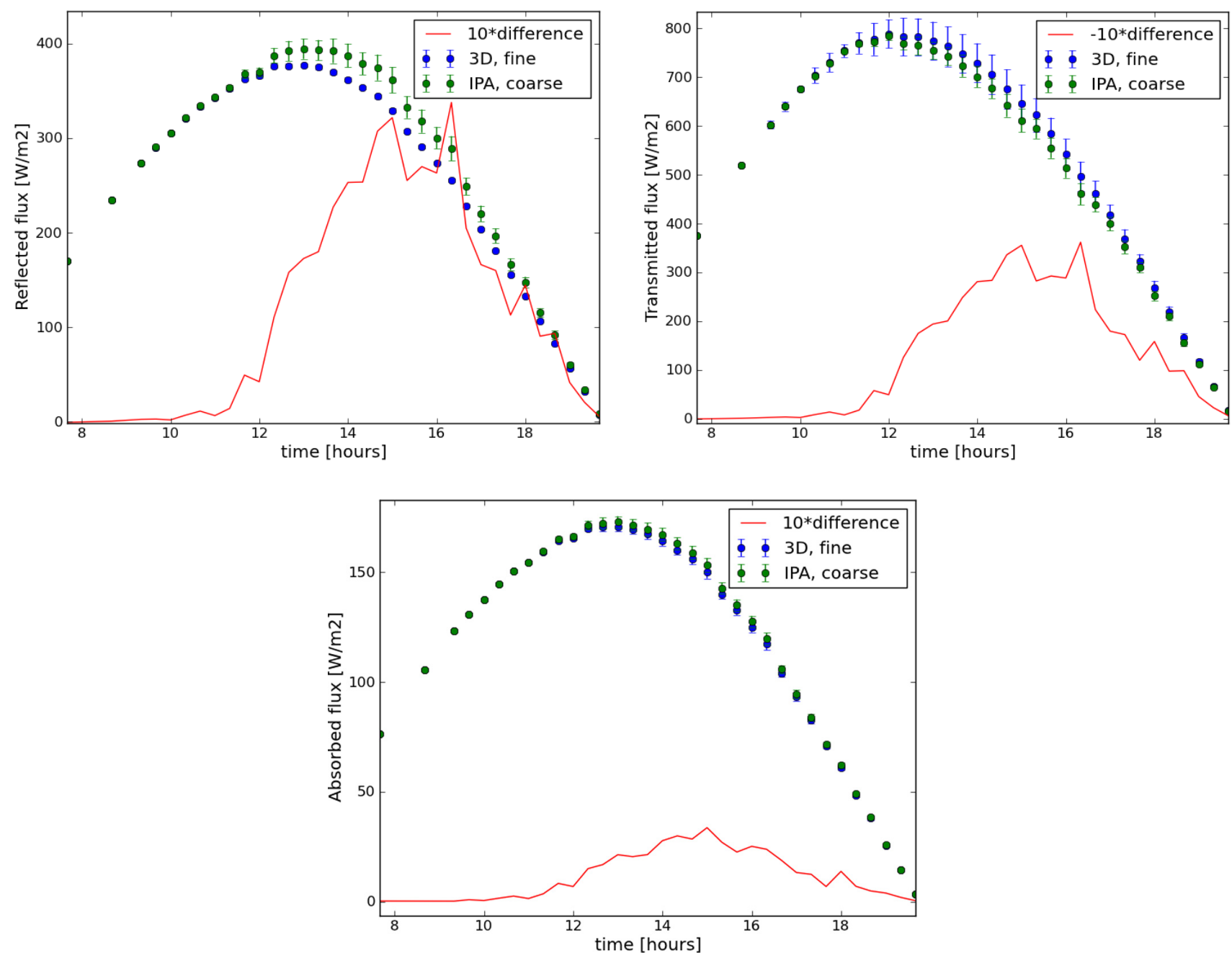

Fig. 8. Radiation fields in a diurnal cycle of shallow cumulus clouds developing over land (see Fig. 3). The left panel shows the reflected flux at the top of the atmosphere, the right top panel, the transmitted flux at ground and the right bottom panel, the absorbed flux through the whole atmosphere. Note that all plots show the shape of the incoming solar radiation which depends on solar zenith angle. The red line represent the bias of the coarse radiation fields in comparison to the high resolution ones. Note that a factor of 10 has been applied to the biases for plotting convenience ( -10 in case of transmitted flux). See text for the explanation of the features.

the coarse fields greatly improves the reflectivity results and eliminate most of the biases.

The neglect of the subscale variability introduces large biases in the radiation fields. For radiation flux densities integrated over the whole solar range, these biases can reach the magnitude of tens of $\mathrm{W} \mathrm{m}^{-2}$ for reflection (albedo) and transmission, and few $\mathrm{W} \mathrm{m}^{-2}$ for absorption. The magnitude of the biases compete with the other main sources of uncertainty in climate and weather prediction model.

Understanding the multi-scale interaction of radiation with heterogeneous cloud fields will help to improve the retrieval of atmospheric constituents. The GMES (Global Monitoring for Environment and Security) satellites will supply upgraded data of the Earth's surface and atmosphere. The quality and quantity of the GMES data will provide a deeper in- sight of the atmosphere, but will also pose some challenges to the processing of the data. In addition to the cloud properties retrieval, which will directly profit from a better description of cloud-radiation interaction, other atmospheric retrievals will also benefit. In particular, the quality of atmospheric gas retrievals greatly depends on the treatment of cloud information, either directly (e.g., cloud fraction and cloud top height) or indirectly (e.g., cloud masking). Hence, a realistic description of clouds will particularly be useful for gas retrievals.

Currently, there are several spacecraft platforms with multiple sensors that provide cloud information at different resolutions. Three of such constellations are MERIS and SCIAMACHY on Envisat (not operative since April 2012); AVHRR, GOME2 and IASI on MetOp; and CAI and TANSO on GOSAT. By means of stochastic methods, cloud 
information at different resolutions can be synergistically combined to create a multi-scale view of the clouds. A better understanding of cloud structure on wide-footprint scenes, will not only imply an enhancement of the quality, but also of the quantity of the data. For instance, in the methane and carbon dioxide retrievals with GOSAT it is tipical to use only cloud-free observations. This reduces the useful data for climate-relevant gases retrieval to ca. $2 \%-5 \%$ of the total.

Additionally, three-dimensional radiative transfer models can supply valuable information to atmospheric observations. On the one hand, 3-D-RT models can be used to estimate the limitations of the one-dimensional theory implemented in the retrieval algorithms. On the other hand, they describe the cloud-radiation interaction more precisely and can provide add-on products (i.e., photon path length statistics) that can help to improve the retrievals.

Acknowledgements. We would like to thank the Intercomparison of Three-dimensional Radiation Codes (I3RC) project for making available their results which we used for the validation of our radiative transfer model MoCaRT. We are specially thankful to Tamás Várnai for his assistance during the results interpretation.

Edited by: A. Kokhanovsky

\section{References}

Barker, H.: A parameterisation for computing grid-averaged solar fluxes for inhomogeneous marine boundary layer clouds. part i: Methodology and homogeneous biases, J. Atmos. Sci., 53, 22892303, 1996.

Barker, H., Goldstein, R., and Stevens, D.: Monte carlo simulation of solar reflectances for cloudy atmospheres, Journal Atmos. Sci., 60, 1881-1894, 2003.

Bar-Or, R. Z., Altaratz, O., and Koren, I.: Global analysis of cloud field coverage and radiative properties, using morphological methods and MODIS observations, Atmos. Chem. Phys., 11, 191-200, doi:10.5194/acp-11-191-2011, 2011.

Brown, A. R., Cederwall, R. T., Chlond, A., Duynkerke, P. G., Golaz, J. C., Khairoutdinov, M., Lewellen, D. C., Lock, A. P., MacVean, M. K., Moeng, C. H., Neggers, R. A. J., Siebesma, A. P., and Stevens, B.: Large-eddy simulation of the diurnal cycle of shallow cumulus convection over land, Q. J. Roy. Meteorol. Soc., 128, 1075-1093, 2002.

Bugliaro, L., Zinner, T., Keil, C., Mayer, B., Hollmann, R., Reuter, M., and Thomas, W.: Validation of cloud property retrievals with simulated satellite radiances: a case study for SEVIRI, Atmos. Chem. Phys., 11, 5603-5624, doi:10.5194/acp-11-5603-2011, 2011.

Buras, R. and Mayer, B.: Efficient unbiased variance reduction techniques for monte carlo simulations of radiative transfer in cloudy atmospheres: The solution, J. Quant. Spectr. Radiat. T., 112, 434447, 2011.

Cahalan, R. F.: Bounded cascade clouds: albedo and effective thickness, Nonlin. Processes Geophys., 1, 156-167, doi:10.5194/npg1-156-1994, 1994.
Cahalan, R., Ridgway, W., Wiscombe, W., and Gollmer, S.: Independent Pixel and Monte Carlo Estimates of Stratocumulus Albedo, J. Atmos. Sci., 51, 3776-3790, 1994.

Cahalan, R. F., Oreopoulos, L., Marshak, A., Evans, E. F., Davis, A. B., Pincus, R., Yetzer, K. H., Mayer, B., Davies, R., Ackerman, T. P., Barker, H. W., Clothiaux, E. E., Ellingson, R. G., Garay, M. J., Kassianov, E., Kinne, S., Macke, A., O’Hirok, W., Partain, P. T., Prigarin, S. M., Rublev, A. N., Stephens, G. L., Szczap, F., Takara, E. E., Vánai, T., Wen, G., and Zhuravleva, T. B.: The i3rc - bringing together the most advanced radiative transfer tools for cloudy atmospheres, B. Am. Meteorol. Soc., 86, 1275-1293, doi:10.1175/BAMS-86-9-1275, 2005.

Cairns, B., Lacis, A. A., and Carlson, B. E.: Absorption within inhomogeneous clouds and its parameterisation in general circulation models, J. Atmos. Sci., 57, 700-714, 2000.

Davis, A. and Marshak, A.: Solar radiation transport in the cloudy atmosphere: a $3 \mathrm{~d}$ perspective on observations and climate impacts, Reports on Progress in Physics, 73, 026801, 2010.

Deirmendjian, D.: Scattering and polarization properties of water clouds and hazes in the visible and infrared, Appl. Optics, 3, 187196, 1964.

Evans, K. F.: The spherical harmonics discrete ordinate method for three-dimensional atmospheric radiative transfer, J. Atmos. Sci., 55, 429-446, 1998.

$\mathrm{Fu}$, Q. and Liou, K. N.: On the correlated k-distribution method for radiative transfer in nonhomogeneous atmospheres, J. Atmos. Sci., 49, 2139-2156, 1992.

Giuseppe, F. D. and Tompkins, A. M.: Effect of spatial organization on solar radiative transfer in three-dimensional idealized stratocumulus cloud fields, J. Atmos. Sci., 60, 1774-1794, 2003.

Henderson-Sellers, A. and Wilson, M.: Surface albedo data for climatic modelling, Rev. Geophys., 21, 1743-1778, 1983.

Hess, M., Koepke, P., and Schult, I.: Optical properties of aerosols and clouds: The software package opac, B. Am. Meteorol. Soc., 79, 831-844, 1998.

Hinkelman, L., Evans, K., Clothiaux, E., Ackerman, T., and Stackhouse Jr., P.: The effect of cumulus cloud field anisotropy on domain-averaged solar fluxes and atmospheric heating rates, J. Atmos. Sci., 64, 3499-3520, 2007.

Jensen, J.: Sur les fonctions convexes et les inégalités entre les valeurs moyennes, Acta Mathematica, 30, 175-193, 1906.

King, M.: Determination of the scaled optical thickness of clouds from reflected solar radiation measurements, J. Atmos. Sci., 44, 1734-1751, 1987.

Kniffka, A. and Trautmann, T.: Combining the independent pixel and point-spread function approaches to simulate the actinic radiation field in moderately inhomogeneous $3 \mathrm{~d}$ cloudy media, $\mathrm{J}$. Quant. Spectr. Radiat. T., 112, 1383-1393, 2011.

Kurucz, R.: The solar spectrum: atlases and line identifications, in: Workshop on Laboratory and Astronomical High resolution Spectra, Astron. Soc. of the Pac. Conf. Ser, 81, 17-31, 1995.

Lovejoy, S., Watson, B., Grosdidier, Y., and Schertzer, D.: Scattering in thick multifractal clouds, part ii: Multiple scattering, Physica A: Statistical Mechanics and its Applications, 388, 37113727, 2009.

Maddux, B., Ackerman, S., and Platnick, S.: Viewing geometry dependencies in modis cloud products, J. Atmos. Oceanic Technol., 27, 1519-1528, 2010. 
Marchuk, G., Mikhailov, G., and Nazaraliev, M.: The Monte Carlo methods in atmospheric optics, Springer Series in Optical Sciences, 1980.

Marshak, A. and Davis, A.: 3D radiative transfer in cloudy atmospheres, Springer, 2005.

Marshak, A., Davis, A., Cahalan, R., and Wiscombe, W.: Nonlocal independent pixel approximation: Direct and inverse problems, Geoscience and Remote Sensing, IEEE Transactions on, 36, 192-205, 1998.

Montopoli, M., Pierdicca, N., and Marzano, F.: Spectral downscaling of integrated water vapour fields from satellite infrared observations, Geoscience and Remote Sensing, IEEE Transactions on, 50, 415-428, 2012.

Nakajima, T. and King, M.: Determination of the optical thickness and effective particle radius of clouds from reflected solar radiation measurements. part i: Theory, J. Atmos. Sci., 47, 1878-1893, 1990.

Nicolet, M.: On the molecular scattering in the terrestrial atmosphere: An empirical formula for its calculation in the homosphere, Planet. Space Sci., 32, 1467-1468, 1984.

O'Hirok, W. and Gautier, C.: A three-dimensional radiative transfer model to investigate the solar radiation within a cloudy atmosphere. part i: Spatial effects, J. Atmos. Sci., 55, 2162-2179, 1998.

Oreopoulos, L. and Davies, R.: Plane parallel albedo biases from satellite observations. part i: Dependence on resolution and other factors, J. Climate, 11, 919-932, 1998.

Rossow, W. and Zhang, Y.-C.: Calculation of surface and top of the atmosphere radiative fluxes from physical quantities based on ISCCP data sets 2. Validation and first results., J. Geophys. Res., 100, 1167-1197, 1995.

Schutgens, N. and Roebeling, R.: Validating the validation: the influence of liquid water distribution in clouds on the intercomparison of satellite and surface observations, J. Atmos. Oceanic Technol., 26, 1457-1474, 2009.
Shettle, E. P. and Fenn, R. W.: Models for the aerosols of the lower atmosphere and the effects of humidity variations on their optical properties, Tech. rep., Air Force Geophysics Lab. Hanscom AFB, MA. Optical Physics Div., 1979.

Slingo, A.: A GCM parameterisation for the shortwave radiative properties of water clouds, J. Atmos. Sci., 46, 1419-1427, 1989.

Stephens, G.: Remote sensing of the lower atmosphere, Oxford University Press, New York, NY, USA, 1994.

Varnai, T. and Davies, R.: Effects of cloud heterogeneities on shortwave radiation: Comparison of cloud-top variability and internal heterogeneity, J. Atmos. Sci., 56, 4206-4224, 1999.

Varnai, T. and Marshak, A.: Observations of three-dimensional radiative effects that influence modis cloud optical thickness retrievals, J. Atmos. Sci., 59, 1607-1618, 2002.

Venema, V., Meyer, S., García, S., Kniffka, A., Simmer, C., Crewell, S., Löhnert, U., Trautmann, T., and Macke, A.: Surrogate cloud fields generated with the iterative amplitude adapted fourier transform algorithm, Tellus A, 58, 104-120, 2006.

Venema, V., Gimeno Garcia, S., and Simmer, C.: A new algorithm for the downscaling of cloud fields, Q. J. Roy. Meteorol. Soc., 136, 91-106, 2010.

Watson, B., Lovejoy, S., Grosdidier, Y., and Schertzer, D.: Scattering in thick multifractal clouds, part $\mathrm{i}$ : Overview and single scattering, Physica A: Statistical Mechanics and its Applications, 388, 3695-3710, 2009.

Zinner, T. and Mayer, B.: Remote sensing of stratocumulus clouds: Uncertainties and biases due to inhomogeneity, J. Geophys. Res., 111, D14209, doi:10.1029/2005JD006955, 2006. 